\title{
Binomial Cubic Fibonacci Sums
}

\author{
Kunle Adegoke \\ Department of Physics and Engineering Physics \\ Obafemi Awolowo University \\ 220005 Ile-Ife, Nigeria \\ adegoke00@gmail.com
}

2010 Mathematics Subject Classification: Primary 11B39; Secondary 11B37.

Keywords: Fibonacci number, Lucas number, summation identity, series, binomial coefficient, cubic Fibonacci identity.

\begin{abstract}
We evaluate some binomial cubic Fibonacci sums.
\end{abstract}

\section{Introduction}

As noted by Nagy et al. [4], there is a paucity of binomial cubic Fibonacci and Lucas identities in existing literature.

Let $F_{j}$ and $L_{j}$ be the $j^{\text {th }}$ Fibonacci number and Lucas number, respectively.

Our main goal is to evaluate the following sums,

$$
\begin{gathered}
\sum_{k=0}^{n}\left(\begin{array}{l}
n \\
k
\end{array}\right) F_{k+s}^{3}, \quad \sum_{k=0}^{n}(-1)^{k}\left(\begin{array}{l}
n \\
k
\end{array}\right) F_{k+s}^{3}, \quad \sum_{k=0}^{n} 2^{k}\left(\begin{array}{l}
n \\
k
\end{array}\right) F_{k+s}^{3}, \\
\sum_{k=0}^{n}(-1)^{k}\left(\begin{array}{l}
n \\
k
\end{array}\right) 2^{n-k} F_{k+s}^{3}, \quad \sum_{k=0}^{n}(-1)^{k}\left(\begin{array}{l}
n \\
k
\end{array}\right) 3^{k} F_{k+s}^{3}, \quad \sum_{k=0}^{n}\left(\begin{array}{l}
n \\
k
\end{array}\right) 3^{n-k} F_{k+s}^{3}, \\
\sum_{k=0}^{\lfloor n / 2\rfloor}\left(\begin{array}{c}
n \\
2 k
\end{array}\right) F_{2 k+s}^{3}, \quad \sum_{k=1}^{\lceil n / 2\rceil}\left(\begin{array}{c}
n \\
2 k-1
\end{array}\right) F_{2 k+s}^{3},
\end{gathered}
$$

and the corresponding series involving Lucas numbers, for any non-negative integer $n$ and any integer $s$.

The Fibonacci numbers, $F_{j}$, and the Lucas numbers, $L_{j}$, are defined, for $j \in \mathbb{Z}$, through the recurrence relations

$$
F_{j}=F_{j-1}+F_{j-2},(j \geq 2), \quad F_{0}=0, F_{1}=1
$$


and

$$
L_{j}=L_{j-1}+L_{j-2},(j \geq 2), \quad L_{0}=2, L_{1}=1
$$

with

$$
F_{-j}=(-1)^{j-1} F_{j}, \quad L_{-j}=(-1)^{j} L_{j} .
$$

Throughout this paper, we denote the golden ratio, $(1+\sqrt{5}) / 2$, by $\alpha$ and write $\beta=(1-$ $\sqrt{5}) / 2=-1 / \alpha$, so that $\alpha \beta=-1$ and $\alpha+\beta=1$.

Explicit formulas (Binet formulas) for the Fibonacci and Lucas numbers are

$$
F_{j}=\frac{\alpha^{j}-\beta^{j}}{\alpha-\beta}, \quad L_{j}=\alpha^{j}+\beta^{j}, \quad j \in \mathbb{Z} .
$$

Koshy [3] and Vajda [6] have written excellent books dealing with Fibonacci and Lucas numbers.

\section{Required identities}

Lemma 1. For real or complex $z$, let a given well-behaved function $h(z)$ have, in its domain, the representation $h(z)=\sum_{k=c_{1}}^{c_{2}} g(k) z^{f(k)}$ where $f(k)$ and $g(k)$ are given real sequences and $c_{1}, c_{2} \in[-\infty, \infty]$. Let $j$ be an integer. Then,

$$
\begin{aligned}
& 5 \sqrt{5} \sum_{k=c_{1}}^{c_{2}} g(k) z^{f(k)} F_{j f(k)}^{3} \\
& \quad=h\left(\alpha^{3 j}\right)-h\left(\beta^{3 j}\right)-3\left(h\left((-1)^{j} \alpha^{j} z\right)-h\left((-1)^{j} \beta^{j} z\right)\right), \\
& \sum_{k=c_{1}}^{c_{2}} g(k) z^{f(k)} L_{j f(k)}^{3} \\
& \quad=h\left(\alpha^{3 j}\right)+h\left(\beta^{3 j}\right)+3\left(h\left((-1)^{j} \alpha^{j} z\right)+h\left((-1)^{j} \beta^{j} z\right)\right) .
\end{aligned}
$$

Proof. Set $m=3$ in Adegoke [1, identities (F) and (L)].

Lemma 2. Let $a, b, c$ and $d$ be rational numbers and $\lambda$ an irrational number. Then,

$$
a+\lambda b=c+\lambda d \Longleftrightarrow a=c, \quad b=d
$$

Lemma 3. For $p$ and $q$ integers,

$$
\begin{gathered}
1+(-1)^{p} \alpha^{2 q}=\left\{\begin{array}{l}
(-1)^{p} \alpha^{q} F_{q} \sqrt{5}, \quad \text { if } p \text { and } q \text { have different parity; } \\
(-1)^{p} \alpha^{q} L_{q}, \quad \text { if } p \text { and } q \text { have the same parity. }
\end{array}\right. \\
1-(-1)^{p} \alpha^{2 q}=\left\{\begin{array}{l}
(-1)^{p-1} \alpha^{q} L_{q}, \text { if } p \text { and } q \text { have different parity; } \\
(-1)^{p-1} \alpha^{q} F_{q} \sqrt{5}, \quad \text { if } p \text { and } q \text { have the same parity. }
\end{array}\right.
\end{gathered}
$$


Proof. We have

$$
\begin{aligned}
(-1)^{p+q}+(-1)^{p} \alpha^{2 q} & =\alpha^{p+q} \beta^{p+q}+\alpha^{p+2 q} \beta^{p} \\
& =\alpha^{p+q} \beta^{p}\left(\alpha^{q}+\beta^{q}\right) \\
& =(-1)^{p} \alpha^{q} L_{q}
\end{aligned}
$$

Similarly,

$$
(-1)^{p+q}-(-1)^{p} \alpha^{2 q}=(-1)^{p-1} \alpha^{q} F_{q} \sqrt{5} .
$$

Corresponding to (4) and (5) we have

$$
(-1)^{p+q}+(-1)^{p} \beta^{2 q}=(-1)^{p} \beta^{q} L_{q}
$$

and

$$
(-1)^{p+q}-(-1)^{p} \beta^{2 q}=(-1)^{p} \beta^{q} F_{q} \sqrt{5} .
$$

Identities (4), (5), (6) and (7) imply

$$
\begin{gathered}
(-1)^{q}+\alpha^{2 q}=\alpha^{q} L_{q} \\
(-1)^{q}-\alpha^{2 q}=-\alpha^{q} F_{q} \sqrt{5} \\
(-1)^{q}+\beta^{2 q}=\beta^{q} L_{q} \\
(-1)^{q}-\beta^{2 q}=\beta^{q} F_{q} \sqrt{5}
\end{gathered}
$$

Lemma 4 (Hoggatt et al [2]). For $p$ and $q$ integers,

$$
\begin{gathered}
L_{p+q}-L_{p} \alpha^{q}=-\beta^{p} F_{q} \sqrt{5} \\
L_{p+q}-L_{p} \beta^{q}=\alpha^{p} F_{q} \sqrt{5} \\
F_{p+q}-F_{p} \alpha^{q}=\beta^{p} F_{q} \\
F_{p+q}-F_{p} \beta^{q}=\alpha^{p} F_{q} .
\end{gathered}
$$

Lemma 5. We have

$$
\begin{gathered}
1-\alpha=\beta, \quad 1-\beta=\alpha, \quad 1+\alpha^{3}=2 \alpha^{2}, \quad 1+\beta^{3}=2 \beta^{2}, \\
1+\alpha=\alpha^{2}, \quad 1+\beta=\beta^{2}, \quad 1-\alpha^{3}=-2 \alpha, \quad 1-\beta^{3}=-2 \beta, \\
1-2 \alpha=-\sqrt{5}, \quad 1-2 \beta=\sqrt{5}, \quad 1+2 \alpha^{3}=\alpha^{3} \sqrt{5}, \quad 1+2 \beta^{3}=-\beta^{3} \sqrt{5}, \\
2+\alpha=\alpha \sqrt{5}, \quad 2+\beta=-\beta \sqrt{5}, \quad 2-\alpha^{3}=-\sqrt{5}, \quad 2-\beta^{3}=\sqrt{5}, \\
1+3 \alpha=\alpha^{2} \sqrt{5}, \quad 1+3 \beta=-\beta^{2} \sqrt{5}, \quad 1-3 \alpha^{3}=-2 \alpha^{2} \sqrt{5}, \quad 1-3 \beta^{3}=2 \beta^{2} \sqrt{5}, \\
3-\alpha=-\beta \sqrt{5}, \quad 3-\beta=\alpha \sqrt{5}, \quad 3+\alpha^{3}=2 \alpha \sqrt{5}, \quad 3+\beta^{3}=-2 \beta \sqrt{5} .
\end{gathered}
$$

Proof. Each identity is obtained by making appropriate substitutions for $p$ and $q$ in the identities given in Lemma 4. 


\section{Binomial cubic Fibonacci identities}

Lemma 6. For non-negative integer $n$, integers $j, r$ and $s$ and real or complex $x$ and $z$,

$$
\begin{aligned}
5 \sqrt{5} \sum_{k=0}^{n}\left(\begin{array}{l}
n \\
k
\end{array}\right) x^{n-k} z^{k} F_{j(r k+s)}^{3}= & \alpha^{3 j s}\left(x+\alpha^{3 j r} z\right)^{n}-\beta^{3 j s}\left(x+\beta^{3 j r} z\right)^{n} \\
- & (-1)^{j s} 3 \alpha^{j s}\left(x+(-1)^{j r} \alpha^{j r} z\right)^{n} \\
& +(-1)^{j s} 3 \beta^{j s}\left(x+(-1)^{j r} \beta^{j r} z\right)^{n}, \\
\sum_{k=0}^{n}\left(\begin{array}{l}
n \\
k
\end{array}\right) x^{n-k} z^{k} L_{j(r k+s)}^{3}=\alpha^{3 j s}( & \left.+\alpha^{3 j r} z\right)^{n}+\beta^{3 j s}\left(x+\beta^{3 j r} z\right)^{n} \\
+ & (-1)^{j s} 3 \alpha^{j s}\left(x+(-1)^{j r} \alpha^{j r} z\right)^{n} \\
+ & (-1)^{j s} 3 \beta^{j s}\left(x+(-1)^{j r} \beta^{j r} z\right)^{n} .
\end{aligned}
$$

Proof. Set $m=3$ in Adegoke [1, identities $\left(\mathrm{BF}^{\prime}\right)$ and $\left.\left(\mathrm{BL}^{\prime}\right)\right]$.

Theorem 1. For non-negative integer $n$ and any integer $s$,

$$
\begin{gathered}
\sum_{k=0}^{n}\left(\begin{array}{l}
n \\
k
\end{array}\right) F_{k+s}^{3}=\frac{1}{5}\left(2^{n} F_{2 n+3 s}+3 F_{n-s}\right), \\
\sum_{k=0}^{n}\left(\begin{array}{l}
n \\
k
\end{array}\right) L_{k+s}^{3}=2^{n} L_{2 n+3 s}+3 L_{n-s},
\end{gathered}
$$

Proof. Set $x=1, z=1, j=1, r=1$ in (F1), utilizing identity (16), to obtain

$$
5 \sqrt{5} \sum_{k=0}^{n}\left(\begin{array}{l}
n \\
k
\end{array}\right) F_{k+s}^{3}=2^{n}\left(\alpha^{3 s+2 n}-\beta^{3 s+2 n}\right)+3\left(\alpha^{n-s}-\beta^{n-s}\right) ;
$$

and hence identity (22). To prove identity $(23)$, use these $(x, z, j, \ldots)$ values in (L1).

The $s=0$ special case of (22) was obtained by Stanica [5].

Theorem 2. For non-negative integer $n$ and any integer $s$,

$$
\begin{gathered}
\sum_{k=0}^{n}\left(\begin{array}{l}
n \\
k
\end{array}\right)(-1)^{k} F_{k+s}^{3}=\frac{1}{5}\left((-1)^{n} 2^{n} F_{n+3 s}-(-1)^{s} 3 F_{2 n+s}\right), \\
\sum_{k=0}^{n}(-1)^{k}\left(\begin{array}{l}
n \\
k
\end{array}\right) L_{k+s}^{3}=(-1)^{n} 2^{n} L_{n+3 s}+(-1)^{s} 3 L_{2 n+s},
\end{gathered}
$$


Proof. To prove identity (24), set $x=1, z=-1, j=1, r=1$ in (F1), noting the identities in (17), to get

$$
5 \sqrt{5} \sum_{k=0}^{n}(-1)^{k}\left(\begin{array}{l}
n \\
k
\end{array}\right) F_{k+s}^{3}=(-1)^{n} 2^{n}\left(\alpha^{n+3 s}-\beta^{n+3 s}\right)-3(-1)^{s}\left(\alpha^{2 n+s}-\beta^{2 n+s}\right),
$$

from which the identity follows. The proof of (25) is similar. Use these values in (L1).

Stanica [5] also found the $s=0$ case of identity (24).

Theorem 3. For non-negative integer $n$ and any integer $s$,

$$
\begin{aligned}
& \sum_{k=0}^{n}\left(\begin{array}{l}
n \\
k
\end{array}\right) 2^{k} F_{k+s}^{3}= \begin{cases}5^{n / 2-1}\left(F_{3 n+3 s}-(-1)^{s} 3 F_{s}\right), & n \text { even } \\
5^{(n-3) / 2}\left(L_{3 n+3 s}+(-1)^{s} 3 L_{s}\right) & n \text { odd },\end{cases} \\
& \sum_{k=0}^{n}\left(\begin{array}{l}
n \\
k
\end{array}\right) 2^{k} L_{k+s}^{3}= \begin{cases}5^{n / 2}\left(L_{3 n+3 s}+(-1)^{s} 3 L_{s}\right), & n \text { even } \\
5^{(n+1) / 2}\left(F_{3 n+3 s}-(-1)^{s} 3 F_{s}\right) & n \text { odd } .\end{cases}
\end{aligned}
$$

Proof. The proof of (26) proceeds with the choice $j=1, r=1, x=1, z=2$ in (F1), employing the set of identities (18), giving

$$
\begin{aligned}
5 \sqrt{5} \sum_{k=0}^{n} 2^{k}\left(\begin{array}{l}
n \\
k
\end{array}\right) F_{k+s}^{3}=( & \sqrt{5})^{n}\left(\alpha^{3 n+3 s}-(-1)^{n} \beta^{3 n+3 s}\right) \\
& -3(-1)^{n+s}(\sqrt{5})^{n}\left(\alpha^{s}-(-1)^{n} \beta^{s}\right),
\end{aligned}
$$

from which the identity follows in accordance with the parity of $n$. The proof of (27) is similar. Use these $(x, z, j, \ldots)$ values in (L1).

Theorem 4. For non-negative integer $n$ and any integer $s$,

$$
\begin{aligned}
& \sum_{k=0}^{n}(-1)^{k}\left(\begin{array}{l}
n \\
k
\end{array}\right) 2^{n-k} F_{k+s}^{3}=\left\{\begin{array}{lr}
5^{n / 2-1}\left((-1)^{s-1} 3 F_{n+s}+F_{3 s}\right), & n \text { even } \\
5^{(n-3) / 2}\left((-1)^{s-1} 3 L_{n+s}-L_{3 s}\right), & n \text { odd }
\end{array}\right. \\
& \sum_{k=0}^{n}(-1)^{k}\left(\begin{array}{l}
n \\
k
\end{array}\right) 2^{n-k} L_{k+s}^{3}=\left\{\begin{array}{l}
5^{n / 2}\left((-1)^{s} 3 L_{n+s}+L_{3 s}\right), \quad n \text { even } ; \\
5^{(n+1) / 2}\left((-1)^{s} 3 F_{n+s}-F_{3 s}\right), \quad n \text { odd. }
\end{array}\right.
\end{aligned}
$$

Proof. The coice $x=2, z=-1, j=1, z=1$ in (F1), noting the set of identities (19) gives

$$
\begin{aligned}
5 \sqrt{5} \sum_{k=0}^{n}(-1)^{k}\left(\begin{array}{l}
n \\
k
\end{array}\right) 2^{n-k} F_{k+s}^{3}= & (\sqrt{5})^{n}(-1)^{n}\left(\alpha^{3 s}-(-1)^{n} \beta^{3 s}\right) \\
& -(\sqrt{5})^{n}(-1)^{s} 3\left(\alpha^{n+s}-(-1)^{n} \beta^{n+s}\right) ;
\end{aligned}
$$

from which we get (28). The proof of (29) is similar. 
Theorem 5. For non-negative integer $n$ and any integer $s$,

$$
\begin{aligned}
& \sum_{k=0}^{n}(-1)^{k}\left(\begin{array}{l}
n \\
k
\end{array}\right) 3^{k} F_{k+s}^{3}=\left\{\begin{array}{l}
5^{n / 2-1}\left(2^{n} F_{2 n+3 s}-(-1)^{s} 3 F_{2 n+s}\right), \quad n \text { even } ; \\
-5^{(n-3) / 2}\left(2^{n} L_{2 n+3 s}+(-1)^{s} 3 L_{2 n+s}\right), \quad n \text { odd } ;
\end{array}\right. \\
& \sum_{k=0}^{n}(-1)^{k}\left(\begin{array}{l}
n \\
k
\end{array}\right) 3^{k} L_{k+s}^{3}=\left\{\begin{array}{l}
5^{n / 2}\left(2^{n} L_{2 n+3 s}+(-1)^{s} 3 L_{2 n+s}\right), \quad n \text { even } ; \\
-5^{(n+1) / 2}\left(2^{n} F_{2 n+3 s}-(-1)^{s} 3 F_{2 n+s}\right), \quad n \text { odd } .
\end{array}\right.
\end{aligned}
$$

Proof. Choose $x=1, z=-3, j=1, r=1$ in (F1). This gives, with the use of the identities in (20),

$$
\begin{aligned}
5 \sqrt{5} \sum_{k=0}^{n}(-1)^{k}\left(\begin{array}{l}
n \\
k
\end{array}\right) 3^{k} F_{k+s}^{3}= & (\sqrt{5})^{n}(-1)^{n} 2^{n}\left(\alpha^{2 n+3 s}-(-1)^{n} \beta^{2 n+3 s}\right) \\
& -(\sqrt{5})^{n}(-1)^{s} 3\left(\alpha^{2 n+s}-(-1)^{n} \beta^{2 n+s}\right) .
\end{aligned}
$$

Identity (30) now follows. The proof of (31) is similar.

Theorem 6. For non-negative integer $n$ and any integer $s$,

$$
\begin{aligned}
& \sum_{k=0}^{n}\left(\begin{array}{l}
n \\
k
\end{array}\right) 3^{n-k} F_{k+s}^{3}= \begin{cases}5^{n / 2-1}\left(2^{n} F_{n+3 s}+3 F_{n-s}\right), & n \text { even } ; \\
5^{(n-3) / 2}\left(2^{n} L_{n+3 s}+3 L_{n-s}\right), & n \text { odd } ;\end{cases} \\
& \sum_{k=0}^{n}\left(\begin{array}{l}
n \\
k
\end{array}\right) 3^{n-k} L_{k+s}^{3}= \begin{cases}5^{n / 2}\left(2^{n} L_{n+3 s}+3 L_{n-s}\right), & n \text { even } ; \\
5^{(n+1) / 2}\left(2^{n} F_{n+3 s}+3 F_{n-s}\right), & n \text { odd } .\end{cases}
\end{aligned}
$$

Proof. Set $x=3, z=1, j=1=r$ in (F1) and use the set of identities in (21) to obtain

$$
\begin{aligned}
5 \sqrt{5} \sum_{k=0}^{n}\left(\begin{array}{l}
n \\
k
\end{array}\right) 3^{n-k} F_{k+s}^{3}= & (\sqrt{5})^{n} 2^{n}\left(\alpha^{n+3 s}-(-1)^{n} \beta^{n+3 s}\right) \\
& +(\sqrt{5})^{n} 3\left(\alpha^{n-s}-(-1)^{n} \beta^{n-s}\right)
\end{aligned}
$$

from which (32) follows. The proof of (33) is similar. Use the same $(x, z, \ldots)$ values in (L1).

Lemma 7. For non-negative integer $n$, integers $j, r$ and $s$ and real or complex $z$,

$$
\begin{aligned}
5 \sqrt{5} \sum_{k=0}^{\lfloor n / 2\rfloor} 2\left(\begin{array}{c}
n \\
2 k
\end{array}\right) z^{2 k} F_{j(2 r k+s)}^{3} & \alpha^{3 j s}\left(1+\alpha^{3 j r} z\right)^{n}+\alpha^{3 j s}\left(1-\alpha^{3 j r} z\right)^{n}-\beta^{3 j s}\left(1+\beta^{3 j r} z\right)^{n}-\beta^{3 j s}\left(1-\beta^{3 j r} z\right)^{n} \\
& \quad-(-1)^{j s} \alpha^{j s} 3\left(1+(-1)^{j r} \alpha^{j r} z\right)^{n}-(-1)^{j s} \alpha^{j s} 3\left(1-(-1)^{j r} \alpha^{j r} z\right)^{n} \\
& \quad+(-1)^{j s} \beta^{j s} 3\left(1+(-1)^{j r} \beta^{j r} z\right)^{n}+(-1)^{j s} \beta^{j s} 3\left(1-(-1)^{j r} \beta^{j r} z\right)^{n},
\end{aligned}
$$




$$
\begin{aligned}
& 2 \sum_{k=0}^{\lfloor n / 2\rfloor}\left(\begin{array}{c}
n \\
2 k
\end{array}\right) z^{2 k} L_{j(2 r k+s)}^{3} \\
& =\alpha^{3 j s}\left(1+\alpha^{3 j r} z\right)^{n}+\alpha^{3 j s}\left(1-\alpha^{3 j r} z\right)^{n}+\beta^{3 j s}\left(1+\beta^{3 j r} z\right)^{n}+\beta^{3 j s}\left(1-\beta^{3 j r} z\right)^{n} \\
& +(-1)^{j s} \alpha^{j s} 3\left(1+(-1)^{j r} \alpha^{j r} z\right)^{n}+(-1)^{j s} \alpha^{j s} 3\left(1-(-1)^{j r} \alpha^{j r} z\right)^{n} \\
& +(-1)^{j s} \beta^{j s} 3\left(1+(-1)^{j r} \beta^{j r} z\right)^{n}+(-1)^{j s} \beta^{j s} 3\left(1-(-1)^{j r} \beta^{j r} z\right)^{n} \text {, } \\
& 5 \sqrt{5} \sum_{k=1}^{\lceil n / 2\rceil} 2\left(\begin{array}{c}
n \\
2 k-1
\end{array}\right) z^{2 k-1} F_{j(2 r k+s)}^{3} \\
& =\alpha^{3 j(r+s)}\left(1+\alpha^{3 j r} z\right)^{n}-\alpha^{3 j(r+s)}\left(1-\alpha^{3 j r} z\right)^{n}-\beta^{3 j(r+s)}\left(1+\beta^{3 j r} z\right)^{n}+\beta^{3 j(r+s)}\left(1-\beta^{3 j r} z\right)^{n} \\
& -(-1)^{j(r+s)} \alpha^{j(r+s)} 3\left(1+(-1)^{j r} \alpha^{j r} z\right)^{n}+(-1)^{j(r+s)} \alpha^{j(r+s)} 3\left(1-(-1)^{j r} \alpha^{j r} z\right)^{n} \\
& +(-1)^{j(r+s)} \beta^{j(r+s)} 3\left(1+(-1)^{j r} \beta^{j r} z\right)^{n}-(-1)^{j(r+s)} \beta^{j(r+s)} 3\left(1-(-1)^{j r} \beta^{j r} z\right)^{n}, \\
& 2 \sum_{k=1}^{\lceil n / 2\rceil}\left(\begin{array}{c}
n \\
2 k-1
\end{array}\right) z^{2 k-1} L_{j(2 r k+s)}^{3} \\
& =\alpha^{3 j(r+s)}\left(1+\alpha^{3 j r} z\right)^{n}-\alpha^{3 j(r+s)}\left(1-\alpha^{3 j r} z\right)^{n}+\beta^{3 j(r+s)}\left(1+\beta^{3 j r} z\right)^{n}-\beta^{3 j(r+s)}\left(1-\beta^{3 j r} z\right)^{n} \\
& +(-1)^{j(r+s)} \alpha^{j(r+s)} 3\left(1+(-1)^{j r} \alpha^{j r} z\right)^{n}-(-1)^{j(r+s)} \alpha^{j(r+s)} 3\left(1-(-1)^{j r} \alpha^{j r} z\right)^{n} \\
& +(-1)^{j(r+s)} \beta^{j(r+s)} 3\left(1+(-1)^{j r} \beta^{j r} z\right)^{n}-(-1)^{j(r+s)} \beta^{j(r+s)} 3\left(1-(-1)^{j r} \beta^{j r} z\right)^{n} .
\end{aligned}
$$

Proof. In the identities

$$
\begin{gathered}
h_{1}(z)=2 \sum_{k=0}^{\lfloor n / 2\rfloor}\left(\begin{array}{c}
n \\
2 k
\end{array}\right) z^{2 r k+s}=z^{s}\left(1+z^{r}\right)^{n}+z^{s}\left(1-z^{r}\right)^{n}, \\
h_{2}(z)=2 \sum_{k=1}^{\lceil n / 2\rceil}\left(\begin{array}{c}
n \\
2 k-1
\end{array}\right) z^{2 r k+s}=z^{r+s}\left(1+z^{r}\right)^{n}-z^{r+s}\left(1-z^{r}\right)^{n},
\end{gathered}
$$

identify

$g(k)=2\left(\begin{array}{c}n \\ 2 k\end{array}\right), \quad f(k)=2 r k+s, \quad c_{1}=0, \quad c_{2}=\lfloor n / 2\rfloor, \quad h(z)=z^{s}\left(1+z^{r}\right)^{n}+z^{s}\left(1-z^{r}\right)^{n}$,

and use these in (F) and (L) to obtain (F2) and (L2). 
Similarly, use of $g(k)=2\left(\begin{array}{c}n \\ 2 k-1\end{array}\right), \quad f(k)=2 r k+s, \quad c_{1}=1, \quad c_{2}=\lceil n / 2\rceil, \quad h(z)=z^{s}\left(1+z^{r}\right)^{n}-z^{s}\left(1-z^{r}\right)^{n}$, in (F) and (L) gives (F3) and (L3).

Theorem 7. For non-negative integer $n$ and any integer $s$,

$$
\begin{aligned}
& 10 \sum_{k=0}^{\lfloor n / 2\rfloor}\left(\begin{array}{c}
n \\
2 k
\end{array}\right) F_{2 k+s}^{3}= 2^{n}\left(F_{2 n+3 s}+(-1)^{n} F_{n+3 s}\right) \\
&- 3(-1)^{s}\left(F_{2 n+s}-(-1)^{s} F_{n-s}\right), \\
& 2 \sum_{k=0}^{\lfloor n / 2\rfloor}\left(\begin{array}{c}
n \\
2 k
\end{array}\right) L_{2 k+s}^{3}=2^{n}\left(L_{2 n+3 s}+(-1)^{n} L_{n+3 s}\right) \\
&+3(-1)^{s}\left(L_{2 n+s}+(-1)^{s} L_{n-s}\right) .
\end{aligned}
$$

Proof. The choice of $z=1=j=r$ in (F2) gives

$$
\begin{aligned}
10 \sqrt{5} \sum_{k=0}^{\lfloor n / 2\rfloor}\left(\begin{array}{c}
n \\
2 k
\end{array}\right) F_{2 k+s}^{3}= & 2^{n}\left(\alpha^{2 n+3 s}-\beta^{2 n+3 s}\right)+(-1)^{n} 2^{n}\left(\alpha^{n+3 s}-\beta^{n+3 s}\right) \\
& +3(-1)^{s}\left(\beta^{s} \alpha^{n}-\alpha^{s} \beta^{n}\right)-3(-1)^{s}\left(\alpha^{2 n+s}-\beta^{2 n+s}\right) ;
\end{aligned}
$$

from which identity (34) follows. The proof of (35) is similar; use $z=1=j=r$ in (L2).

Corollary 8. For non-negative integer $n$ and any integer $s$,

$$
\begin{gathered}
10 \sum_{k=0}^{n}\left(\begin{array}{l}
2 n \\
2 k
\end{array}\right) F_{2 k+s}^{3}= \begin{cases}4^{n} L_{n} F_{3 n+3 s}-(-1)^{s} 3 F_{n+s} L_{3 n}, & n \text { even } ; \\
4^{n} F_{n} L_{3 n+3 s}-(-1)^{s} 3 L_{n+s} F_{3 n}, & n \text { odd } ;\end{cases} \\
2 \sum_{k=0}^{n}\left(\begin{array}{l}
2 n \\
2 k
\end{array}\right) L_{2 k+s}^{3}= \begin{cases}4^{n} L_{n} L_{3 n+3 s}+(-1)^{s} 3 L_{n+s} L_{3 n}, & n \text { even } ; \\
5\left(4^{n} F_{n} F_{3 n+3 s}+(-1)^{s} 3 F_{n+s} F_{3 n}\right), & n \text { odd } .\end{cases}
\end{gathered}
$$

Proof. Write $2 n$ for $n$ in each of the identities (34) and (35). Simplification is achieved by the use of the following well-known Fibonacci identities which are valid for any two integers $u$ and $v$ having the same parity:

$$
\begin{aligned}
F_{u}+(-1)^{(u-v) / 2} F_{v} & =L_{(u-v) / 2} F_{(u+v) / 2}, \\
F_{u}-(-1)^{(u-v) / 2} F_{v} & =F_{(u-v) / 2} L_{(u+v) / 2}, \\
L_{u}+(-1)^{(u-v) / 2} L_{v} & =L_{(u-v) / 2} L_{(u+v) / 2}, \\
L_{u}-(-1)^{(u-v) / 2} L_{v} & =5 F_{(u-v) / 2} F_{(u+v) / 2} .
\end{aligned}
$$


Corollary 9. For non-negative integer $n$,

$$
\begin{gathered}
10 \sum_{k=0}^{n}\left(\begin{array}{c}
2 n-1 \\
2 k
\end{array}\right) F_{2 k}^{3}=\left\{\begin{array}{l}
\left(2^{2 n-1}-3\right) F_{2 n-1} L_{n-1} L_{n}, \quad n \text { even } \\
\left(2^{2 n-1}-3\right) 5 F_{2 n-1} F_{n-1} F_{n}, \quad n \text { odd }
\end{array}\right. \\
2 \sum_{k=0}^{n}\left(\begin{array}{l}
2 n \\
2 k
\end{array}\right) L_{2 k}^{3}=\left\{\begin{array}{l}
\left(4^{n}+3\right) L_{n} L_{3 n}, \quad n \text { even } ; \\
\left(4^{n}+3\right) 5 F_{n} F_{3 n}, \quad n \text { odd } .
\end{array}\right.
\end{gathered}
$$

Proof. To prove (42), write $2 n-1$ for $n$ in (34) and set $s=0$. To prove (43), set $s=0$ in identity (37).

Theorem 10. For non-negative integer $n$ and any integer $s$,

$$
\begin{aligned}
10 \sum_{k=1}^{\lceil n / 2\rceil}\left(\begin{array}{c}
n \\
2 k-1
\end{array}\right) F_{2 k+s}^{3}= & 2^{n}\left(F_{2 n+3 s+3}-(-1)^{n} F_{n+3 s+3}\right) \\
& -(-1)^{s} 3\left(F_{2 n+s+1}-(-1)^{s} F_{n-s-1}\right), \\
2 \sum_{k=1}^{\lceil n / 2\rceil}\left(\begin{array}{c}
n \\
2 k-1
\end{array}\right) L_{2 k+s}^{3}= & 2^{n}\left(L_{2 n+3 s+3}-(-1)^{n} L_{n+3 s+3}\right) \\
& +(-1)^{s} 3\left(L_{2 n+s+1}+(-1)^{s} L_{n-s-1}\right) .
\end{aligned}
$$

Proof. Set $z=1=j=r$ in identity (F3) to obtain

$$
\begin{aligned}
10 \sqrt{5} & \sum_{k=1}^{\lceil n / 2\rceil}\left(\begin{array}{c}
n \\
2 k-1
\end{array}\right) F_{2 k+s}^{3} \\
= & 2^{n}\left(\alpha^{2 n+3 s+3}-\beta^{2 n+3 s+3}\right)-(-1)^{n} 2^{n}\left(\alpha^{n+3 s+3}-\beta^{n+3 s+3}\right) \\
& \quad+(-1)^{s+1} 3\left(\alpha^{2 n+s+1}-\beta^{2 n+s+1}\right)+(-1)^{s+1} 3\left(\alpha^{n} \beta^{s+1}-\alpha^{s+1} \beta^{n}\right)
\end{aligned}
$$

from which identity (44) follows. The proof of (45) is similar.

Corollary 11. For non-negative integer $n$ and any integer $s$,

$$
\begin{gathered}
10 \sum_{k=1}^{n}\left(\begin{array}{c}
2 n \\
2 k-1
\end{array}\right) F_{2 k+s}^{3}= \begin{cases}4^{n} F_{n} L_{3 n+3 s+3}-(-1)^{s} 3 L_{n+s+1} F_{3 n}, & n \text { even } \\
4^{n} L_{n} F_{3 n+3 s+3}-(-1)^{s} 3 F_{n+s+1} L_{3 n}, & n \text { odd } ;\end{cases} \\
2 \sum_{k=1}^{n}\left(\begin{array}{c}
2 n \\
2 k-1
\end{array}\right) L_{2 k+s}^{3}= \begin{cases}5\left(4^{n} F_{n} F_{3 n+3 s+3}+(-1)^{s} 3 F_{n+s+1} F_{3 n}\right), & n \text { even } \\
4^{n} L_{n} L_{3 n+3 s+3}+(-1)^{s} 3 L_{n+s+1} L_{3 n}, & n \text { odd } .\end{cases}
\end{gathered}
$$

Proof. Write $2 n$ for $n$ in each of the identities (44) and (45) and make use of identities $(38)-(41)$. 
Corollary 12. For non-negative integer $n$,

$$
\begin{gathered}
10 \sum_{k=1}^{n}\left(\begin{array}{c}
2 n-1 \\
2 k-1
\end{array}\right) F_{2 k-1}^{3}=\left\{\begin{array}{l}
\left(2^{2 n-1}+3\right) 5 F_{2 n-1} F_{n-1} F_{n}, \quad n \text { even } \\
\left(2^{2 n-1}+3\right) F_{2 n-1} L_{n-1} L_{n}, \quad n \text { odd },
\end{array}\right. \\
2 \sum_{k=1}^{n}\left(\begin{array}{c}
2 n \\
2 k-1
\end{array}\right) L_{2 k-1}^{3}= \begin{cases}\left(4^{n}-3\right) 5 F_{n} F_{3 n}, & n \text { even } ; \\
\left(4^{n}-3\right) L_{n} L_{3 n}, & n \text { odd } .\end{cases}
\end{gathered}
$$

\section{References}

[1] K. Adegoke, Binomial Fibonacci power sums, preprint, 2021, doi: 10.20944/preprints202105.0378.v1

[2] V. E. Hoggatt, Jr., J. W. Phillips and H. T. Leonard, Jr., Twenty-four master identities, The Fibonacci Quarterly 9:1 (1971), 1-17.

[3] T. Koshy, Fibonacci and Lucas Numbers with Applications, Wiley-Interscience, 2001.

[4] M. Nagy, S. R. Cowel and V. Beiu, Survey of cubic Fibonacci identities when cuboids carry weight, preprint, 2019, arXiv: 1902.05944v1.

[5] P. Stănică, Generating functions, weighted and non-weighted sums for powers of secondorder recurrence sequences, The Fibonacci Quarterly 41:4 (2003), 321-333.

[6] S. Vajda, Fibonacci and Lucas Numbers, and the Golden Section: Theory and Applications, Dover Press, 2008.

Concerned with sequences: A000032, A000045. 